\title{
Editorial. Implicaciones normativas de la política de aseguramiento de la calidad de la educación superior en Colombia
}

Citation: Rivera Aya, E. N. (2022). Editorial.

Implicaciones normativas de la política de aseguramiento de la calidad de la educación superior en Colombia. Mutis, 12(1).

https://doi.org/10.21789/22 561498.1831

Copyright: () 2022 por los autores.

Licenciado para Mutis. Este artículo es un artículo de acceso abierto distribuido bajo los términos y condiciones de la licencia Creative Commons Attribution (https:// https://creativecommons.org /licenses/by-nc-sa/4.0/).

\author{
Eliasib Naher Rivera Aya ad \\ a Ingeniero industrial, administrador de empresas, magíster en Administración y magíster en Educación. \\ Profesor Asociado de la Facultad de Ciencias Naturales e Ingeniería de la Universidad Jorge Tadeo Lozano, Colombia \\ https://orcid.org/0000-0002-5875-1080
}

En la Constitución de 1991 se establece que el Estado debe velar por la calidad de la educación, y en el artículo 53 de la Ley 30 de 1992 se ordena la creación del Sistema Nacional de Acreditación, el cual, según se estipula en el artículo 54 de la misma ley, contará con un Consejo Nacional de Acreditación. Su objetivo fundamental es "garantizar a la sociedad que las instituciones que hacen parte del Sistema cumplen con los más altos requisitos de calidad y que realizan sus propósitos y objetivos" (CNA, s. f.). Los primeros programas académicos en ser acreditados por el MEN, a partir del concepto del CNA, recibieron este reconocimiento en 1998, lo que indica que el CNA lleva 23 años desarrollando la labor de verificación de las condiciones de alta calidad de programas e instituciones de educación superior en Colombia.

La coyuntura actual relacionada con la política de calidad de la educación superior refleja unos ajustes que se han aplicado a dicha política. Prueba de ello puede verse en la publicación, por parte del Ministerio de Educación Nacional, del Acuerdo 02 de 2020 del CESU, el Decreto 1330 de 2019 y la Resolución 21795 de 2020.

La política de aseguramiento de la calidad de la educación superior en Colombia exige a las universidades obtener el Registro Calificado para cada uno de los programas ofrecidos, es decir, es requisito obligatorio para que los programas puedan funcionar. Esta evaluación la realiza la Comisión Nacional Intersectorial de Aseguramiento de la Calidad de la Educación Superior (CONACES). Pero también está la posibilidad de que las universidades obtengan acreditación de alta calidad para sus programas, así como la acreditación institucional. Esta acreditación (ya sea de programas o institucional) es voluntaria e implica que la institución decide llevar a cabo un proceso de autoevaluación orientado a identificar si, a pesar de las debilidades que pueda tener, sus fortalezas son lo suficientemente sólidas para demostrar ante el Consejo Nacional de Acreditación (CNA) - entidad que se encarga de hacer la evaluaciónque la calidad que ofrece está por encima de la media de los programas similares de otras instituciones. Y si lo que busca es la acreditación institucional de alta calidad, deberá demostrar que la calidad de la formación que ofrece está por encima de la media de las demás instituciones de educación superior. Tanto CONACES como el CNA tenían cada uno un modelo de evaluación para verificar: en el primer caso, el cumplimiento de unos estándares mínimos de calidad, y en el segundo, para verificar el cumplimiento de altos estándares de calidad.

Una dificultad que pedía ser revisada por parte de representantes de las instituciones de educación superior en Colombia era la falta de articulación entre los lineamientos para obtener el Registro Calificado y los lineamientos para obtener la acreditación de alta calidad de programas y universidades. En otras palabras, se solicitaba que los lineamientos requeridos por 
parte de CONACES para obtener el Registro Calificado fueran similares a los lineamientos del CNA para evaluar la alta calidad de programas e instituciones, pero con un mayor grado de exigencia para obtener la acreditación de alta calidad. Esto, porque la diferencia en los modelos de cada uno de estos dos organismos causaba gran desgaste de las instituciones para cumplir con ambos modelos. En cambio, articular los lineamientos ayudaría a elevar la eficiencia en el uso del tiempo destinado a los procesos de autoevaluación de programas e instituciones y era un aliciente para hacer esfuerzos orientados a elevar la calidad ofrecida.

Con el Decreto 1330 de 2019 se establecieron las condiciones de calidad institucionales y de programa para Registro Calificado, en tanto que el Acuerdo 02 del 2020 del Consejo Nacional de Educación Superior (CESU) actualiza el modelo de acreditación de alta calidad de programas e instituciones. Los lineamientos de ambos modelos están ahora articulados, dando así respuesta a la solicitud de las distintas Instituciones de Educación Superior (IES) al respecto. Adicionalmente, el Acuerdo 02 del 2020 busca fortalecer los Sistemas Internos de Aseguramiento de la Calidad de las IES en Colombia, al tiempo que incluye como aspecto importante de la calidad la evaluación de los resultados de aprendizaje de los estudiantes.

Estos ajustes a la normatividad de la política de calidad de la educación superior abren una ventana de oportunidades de investigación en temas como la evaluación de los resultados de aprendizaje de los estudiantes, los efectos hacia futuro que tendrán los cambios normativos en la política de calidad de las instituciones de educación superior, las alternativas estratégicas para fortalecer los sistemas internos de aseguramiento de la calidad de las IES en Colombia, entre otros. Respecto de este último, una línea de investigación puede estar orientada a proponer un modelo o una metodología de toma de decisiones estratégicas en el interior de los sistemas internos de aseguramiento de la calidad que ayude a articular las decisiones estratégicas de las universidades con los ajustes recientes a la normatividad del sistema de aseguramiento de la calidad de la educación superior, así como con los aspectos estratégicos expresados en los Proyectos Educativos Institucionales y en los Planes de Desarrollo de las instituciones de educación superior. Dos de los beneficios de proponer alguna metodología o modelo de toma de decisiones que ayude a dicha articulación son, por una parte, que las IES acreditadas pueden tomar como referencia ese modelo para mejorar aún más sus prácticas en el interior de sus sistemas de aseguramiento de la calidad, dentro de la lógica del mejoramiento continuo que soporta el modelo del CNA, y por otra, que las IES que no están acreditadas tengan un referente que les permita orientar de manera estratégica sus esfuerzos para iniciar o continuar con la autoevaluación con fines de acreditación institucional o de programas.

Adicionalmente, vale la pena tener en cuenta que "las universidades deben pensarse estratégicamente debido especialmente a su entorno cada vez más complejo y cambiante. Asimismo, dado que las funciones relacionadas con la docencia y la investigación se hacen cada vez más diversas y difíciles de abordar sobre la base de intuiciones o de solo decisiones políticas, deben ser ahora objeto de estudio sistemático y de recomendaciones fundamentadas" (Rodríguez Ponce, 2005).

Por otra parte, cabe advertir que "es frecuente que en las organizaciones actuales el desempeño individual sea opuesto al desempeño estratégico de la organización. Por lo tanto, [...] se requiere alinear la evaluación del desempeño individual con la evaluación del desempeño organizacional estratégico para así mantener o mejorar la productividad. Asimismo, las personas encargadas de los niveles de gestión deben tener la capacidad y la visión para alinear adecuadamente a los stakeholders con los objetivos, procesos y estrategias de la organización" (Bautista Herrera, 2017). Esto aplica también para las instituciones de educación superior, cuya estructura organizacional debe evidenciar tanto la división del trabajo como su integración y coherencia con la misión y la visión. 
Dado lo anterior, sería útil para el personal del área de aseguramiento de la calidad de las IES contar con un modelo que ofrezca un marco de reflexión al momento de tomar decisiones sistémicas y alineadas, tanto con la estrategia como con la política de aseguramiento de la calidad de la educación superior propuesta por el Ministerio de Educación Nacional y las demás entidades relacionadas.

\section{REFERENCIAS}

Bautista Herrera, W. (2017). Modelo de alineamiento estratégico para la Facultad de Ingeniería de la Universidad Incca de Colombia [trabajo de grado de maestría]. Bogotá: Universidad Nacional Abierta y a Distancia (UNAD), Maestría en Administración de Organizaciones. https://repository.unad.edu.co/handle/10596/13755

Consejo Nacional de Acreditacipm (CNA. (s.f.). Consejo Nacional de Acreditación. (M. d. Nacional, Editor) Recuperado el 25 de noviembre de 2018, de https://www.cna.gov.co/1741/article-186382.html

Rodríguez Ponce, E. (2005). La toma de decisiones estratégicas en las instituciones universitarias: un estudio empírico en Chile y España. Arica, Chile: Ediciones Universidad de Tarapacá.

https://www.researchgate.net/publication/26520839_El_proceso_de_toma_de_decisiones_est rategicas_en_las_universidades_publicas 